Could dark matter or neutrinos discriminate between the enantiomers of a chiral molecule?

This article has been downloaded from IOPscience. Please scroll down to see the full text article.

2008 EPL 8213002

(http://iopscience.iop.org/0295-5075/82/1/13002)

View the table of contents for this issue, or go to the journal homepage for more

Download details:

IP Address: 147.96.14.15

The article was downloaded on 04/02/2013 at 16:47

Please note that terms and conditions apply. 


\title{
Could dark matter or neutrinos discriminate between the enantiomers of a chiral molecule?
}

\author{
P. Bargueño ${ }^{1,2(a)}$, A. Dobado ${ }^{3}$ and I. Gonzalo ${ }^{4}$ \\ ${ }^{1}$ Instituto de Matemáticas y Física Fundamental (CSIC) - Serrano 123, 20006 Madrid, Spain, EU \\ ${ }^{2}$ Departamento de Química Física, Universidad de Salamanca - 37008 Salamanca, Spain, EU \\ ${ }^{3}$ Departamento de Fúsica Teórica I, Universidad Complutense de Madrid - 28040 Madrid, Spain, EU \\ ${ }^{4}$ Departamento de Óptica, Universidad Complutense de Madrid - 28040 Madrid, Spain, EU
}

received 10 December 2007; accepted in final form 4 February 2008

published online 5 March 2008

PACS 33.15.Bh - General molecular conformation and symmetry; stereochemistry

PACS 13.15.+g - Neutrino interactions

PACS 95.35.+d - Dark matter (stellar, interstellar, galactic, and cosmological)

\begin{abstract}
We examine the effect of cold dark matter on the discrimination between the two enantiomers of a chiral molecule. We estimate the energy difference between the two enantiomers due to the interaction between fermionic WIMPs (weak interacting massive particles) and molecular electrons on the basis that electrons have opposite helicities in opposite enantiomers. It is found that this energy difference is completely negligible. Dark matter could then be discarded as an inductor of chiroselection between enantiomers and then of biological homochirality. However, the effect of cosmological neutrinos, revisited with the currently accepted neutrino density, would reach, in the most favorable case, an upper bound of the same order of magnitude as the energy difference obtained from the well-known electroweak electron-nucleus interaction in some molecules.
\end{abstract}

Copyright (C) EPLA, 2008

Introduction. - The origin of biological homochirality, that is, the almost exclusive one-handedness of chiral molecules in biological organisms, is a fundamental problem for which there is not yet a convincing solution. Several mechanisms have been proposed to explain chiroselection among the two possible enantiomers of a chiral molecule (see, for example, [1-3] and references therein). These mechanisms involve chance, $\beta$-radiolysis [4], circularly polarized light [5-7], magnetic fields [8,9], and violation of parity in the weak interaction (see below).

The discovery of an excess of L-amino acids in meteorites $[10,11]$ has reinforced the idea of an extraterrestrial origin of biological homochirality $[1,12]$. In this context, universal mechanisms of chiroselection such as parity violation in weak interactions would acquire special interest in spite of their tiny effects, without, of course, underestimating other mechanisms.

The effect of electroweak interactions between electrons and nuclei mediated by the $Z^{0}$ have been extensively studied and observed in atoms (see the review [13]), and only predicted in molecules, where an energy difference between the two enantiomers of chiral molecules has been

(a) E-mail: pbdr@imaff.cfmac.csic.es estimated to be between $10^{-16}$ and $10^{-21} \mathrm{eV}[14-18]$. In the laboratory, no conclusive energy difference has been reported in experimental spectroscopic studies reaching an energy resolution of about $10^{-15} \mathrm{eV}$ [19].

The above tiny energy difference would require a powerful mechanism of amplification in order to induce a real enantioselective effect. Otherwise the small energy difference would be masked by the natural broadening of the energy levels of the molecule, thermal fluctuations and environment interactions, which do not discriminate, in average, between $L$ and $D$ enantiomers. There is active research on amplification mechanisms in which a permanent although very small interaction acting always in the same enantioselective direction, and under appropriate conditions, could lead to an effective enantioselection. Some mechanisms are based on nonlinear autocatalytic processes of polymerization or crystallization along a large period of time [20,21]. Another one involves a secondorder phase transition below a certain critical temperature [22] that could work at low temperatures such as those of the interstellar space. However, theoretical or experimental conclusive results from the diverse mechanisms to amplify enantioselection based on electroweak energy difference, are not yet at hand (e.g., $[23,24])$. 
Another universal mechanism that could discriminate between the two enantiomers of a chiral molecule and that involves weak interaction is neutrino-electron axial-vector interaction mediated by charged electroweak bosons $W^{ \pm}$. This process discriminates on the basis of an asymmetry between the number of neutrinos and antineutrinos, and on the fact that electrons of opposite $(\mathrm{L}, \mathrm{R})$ enantiomers have opposite helicity. Considering the cosmological relic sea of neutrinos, the estimated energy difference between the two enantiomers was found to be even lower than the value $10^{-21} \mathrm{eV}$ mentioned above [25]. However, it could increase significantly under bigger neutrino fluxes, as in supernova remnants [26] assumed that big molecules could survive in the surroundings. Revisited assumptions about the number density of cosmological neutrinos in the relic sea lead to an increase of the mentioned energy difference, as we shall see in the next section.

Looking for other universal mechanisms acting also outside of the Earth, we analyze here the possible enantioselective effect of chiral dark matter on chiral molecules.

The existence of dark matter is inferred from astrophysical observations in light of studies of the dynamics of stars in the local disk environment, rotation curves for a large number of spiral galaxies, gravitational lensing by clusters of galaxies and some large-scale studies of the Universe (for a recent review of experimental searches for dark matter see, for example, [27]). A vast variety of candidates has been proposed for dark-matter content, from baryonic to non-baryonic matter. The non-baryonic candidates are basically postulated elementary particles beyond the standard model which have not been discovered yet, like axions, WIMPs (Weak Interacting Massive Particles) and other exotic candidates. The baryonic candidates are the Massive Compact Halo Objects (Macho) [28]. Another important difference is the hot vs. cold dark matter. A dark-matter candidate is called hot if it moves at relativistic speeds at the time when galaxies could just start to form, and cold if it moves non-relativistically at that time. The problem is that hot dark matter cannot reproduce correctly the observed structure of the Universe. Therefore, we focus our attention on cold dark matter. The fact that dark matter interacts weakly with matter makes its detection very difficult [27]. However, many experiments are currently in progress in order to reach this goal.

Here we estimate the energy difference between the two enantiomers of a chiral molecule, due to the weaktype interaction between non-baryonic cold dark matter (specifically WIMPs) and molecular electrons with nonzero helicity. Experimental results on dark matter are used. Given the resemblance in the procedure with the energy difference induced by cosmological neutrinos, estimated in a previous work [25], we first recall this procedure at the time we improve the result we obtained in that work.

Energy difference between opposite enantiomers induced by cosmological neutrinos. - Following a previous work [25], we consider neutrino-electron interactions mediated by the axial-vector Hamiltonian density,

$$
H=\frac{G_{\mathrm{F}}}{\sqrt{2}} \bar{e} \gamma^{\mu}\left(g_{V}-g_{A} \gamma_{5}\right) e \bar{\nu} \gamma_{\mu}\left(1-\gamma_{5}\right) \nu
$$

where $G_{\mathrm{F}}$ is Fermi's constant, $e(\nu)$ denotes the electron (neutrino) spinor field, $\bar{e}(\bar{\nu})$ is its adjoint spinor, $\gamma^{\mu}$ are the Dirac matrices (regarded as a four-vector), $\gamma^{5}=$ $i \gamma^{0} \gamma^{1} \gamma^{2} \gamma^{3}$, and $g_{V, A}$ are suitable coupling constants that parameterize the strength of the interactions. As was discussed for example in [29], in the non-relativistic limit it is possible to make the following approximations for the dominant temporal components of the four-vectors appearing in the above Hamiltonian:

$$
\begin{aligned}
\bar{e} \gamma^{\mu} \gamma_{5} e & \sim \vec{\sigma}_{e} \cdot \vec{v}_{e} & & \\
\bar{\nu} \gamma_{\mu} \nu & \sim n_{\nu}-n_{\bar{\nu}} & & \text { (Dirac neutrinos) } \\
\bar{\nu} \gamma_{\mu} \gamma_{5} \nu & \sim n_{\nu_{l}}-n_{\nu_{r}} & & \text { (Majorana neutrinos) }
\end{aligned}
$$

where the number density differences, $n_{\nu}-n_{\bar{\nu}}$ and $n_{\nu_{l}}-$ $n_{\nu_{r}}$ refer to neutrino-antineutrino and left-right helicity eigenstates, respectively. Obviously they are not zero only in the case where there is a net lepton number or helicity in the cosmic neutrinos background. We recall from [25] and [30] that for Dirac neutrinos the energy splitting obtained for the electron is

$$
\Delta E \sim G_{\mathrm{F}}\left|\left(n_{\nu}-n_{\bar{\nu}}\right)\left\langle\vec{\sigma}_{e} \cdot \vec{v}_{e}\right\rangle\right|,
$$

where the expected value of the electron helicity $\left\langle\vec{\sigma}_{e} \cdot \vec{v}_{e}\right\rangle$ takes opposite signs for the two opposite enantiomers, as we can see from a simplified chiral molecule model [31]. In this model, a dominant axial symmetry around axis $Z$, with a left(right)-handed perturbative potential of period $a$, is assumed, so that the electronic molecular states can be described by superposition of eigenstates of both angular momentum $L_{z}$ and linear momentum $P_{z}$ (eigenvalues $\hbar n(2 \pi / a))$, i.e. $\left|M_{L}, n\right\rangle$, in the form

$$
\begin{gathered}
\Phi_{L}=c_{0}|0,0\rangle+c_{1}|+1,-1\rangle+c_{2}|-1,+1\rangle, \\
\Phi_{R}=c_{0}|0,0\rangle+c_{1}|+1,+1\rangle+c_{2}|-1,-1\rangle
\end{gathered}
$$

with $\left|c_{1}\right|^{2}=\left|c_{2}\right|^{2} \equiv C$. These $L$ and $R$ states have then opposite helicities:

$$
\left\langle\Phi_{L}\left|L_{z} P_{z}\right| \Phi_{L}\right\rangle=-4 C \pi / a=-\left\langle\Phi_{R}\left|L_{z} P_{z}\right| \Phi_{R}\right\rangle .
$$

Notice that we are using all the time natural units where $\hbar=c=1$. The spin of the electron can be taken into consideration by replacing $L_{z}$ with $J_{z}=L_{z}+S_{z}$. In a realistic chiral molecule the electronic states would not be eigenstates of the helicity, but its mean value would have opposite sign for $L$ and $R$ enantiomers. The parameter $C<1 / 2$ accounts for the degree of chirality.

We note that the velocity of the molecule carrier (interstellar grains, meteorites, the Earth...) does not contribute to the helicity of the electrons: If $\vec{P}_{T}$ is the 
translational momentum of the carrier, the electronic wave function $\Phi_{L(R)}$ must include the factor $e^{i \vec{P}_{T} \cdot \vec{R}}$ (here $\vec{R}$ is the position of the molecule), and the contribution of $\vec{P}_{T}$ to the electron helicity is then

$$
\begin{aligned}
& \left\langle\Phi_{L(R)} e^{i \vec{P}_{T} \cdot \vec{R}}\left|\vec{L} \cdot \vec{P}_{T}\right| \Phi_{L(R)} e^{i \vec{P}_{T} \cdot \vec{R}}\right\rangle= \\
& \left\langle\Phi_{L(R)}|\vec{L}| \Phi_{L(R)}\right\rangle \cdot \vec{P}_{T}=0,
\end{aligned}
$$

since $\left\langle\Phi_{L}|\vec{L}| \Phi_{L}\right\rangle=\left\langle\Phi_{R}|\vec{L}| \Phi_{R}\right\rangle=0$ as can be seen from eqs. (4), (5). We also remark that the particle flux is assumed to be isotropic, thus, its interaction with the electrons of a chiral molecule is the same irrespective of the orientation of the molecule.

The energy difference that we obtained, assuming complete neutrino-antineutrino asymmetry, with number density of about $10^{-2} \mathrm{~cm}^{-3}, C=1 / 2, a \sim 1$ Ångström and the electron helicity given by eq. (6), was of the order of $10^{-26} \mathrm{eV}$ [25].

However, it has been recently suggested $[29,32]$ that, in scenarios beyond the standard model, the neutrinoantineutrino density asymmetry $n_{\nu}-n_{\bar{\nu}}$ could be up to the order of $\sim 10-1050 \mathrm{~cm}^{-3}$. Although the extreme upper-bound density asymmetry seems to be excluded by considerations of primordial nucleosynthesis [33], we consider it to estimate an upper bound of the energy difference.

If we take the value $n_{\nu}-n_{\bar{\nu}} \sim 1000 \mathrm{~cm}^{-3}$, we then obtain an upper bound for the energy difference between enantiomers of the order of $10^{-21} \mathrm{eV}$, per molecular electron with non-zero helicity. Evidently this tiny energy needs massive amplification mechanisms as those mentioned at the beginning in order to induce an effective enantioselection.

Energy difference between opposite enantiomers induced by fermionic cold dark matter. - In a way similar to the neutrino-electron interaction considered above, we are now to estimate the electron energy splitting induced by the axial-vector interaction between a fermionic dark-matter candidate (typically a WIMP) and an electron. The relevant Hamiltonian density can be written as

$$
H=\sum_{i} d_{i} \bar{\chi} \gamma_{\mu}\left(1-\gamma_{5}\right) \chi \bar{\psi}_{i} \gamma^{\mu} \gamma_{5} \psi_{i}
$$

where $\chi$ is the dark-matter spinor which can be a Dirac or a Majorana one. The index $i$ runs through $i=e, u, d, s$, i.e., we are considering also the interaction between the dark-matter particle and the $u, d$ and $s$ quarks. This will be important later in order to use the present experiments trying to measure the WIMP flux on Earth to set some bounds on the possible effect of dark matter on opposite enantiomers. Therefore, $d_{e}, d_{u}, d_{d}$ and $d_{s}$ are the coupling of the $\chi$ field to the different matter fields $e=\psi_{e}, u=\psi_{u}, d=\psi_{d}$ and $s=\psi_{s}$.

As WIMPs are typical examples of cold dark matter and heavy by definition, we can invoke again the nonrelativistic limit. Thus, as it was the case of neutrinos, for Dirac WIMPs the term $\bar{\chi} \gamma_{\mu} \chi$ dominates with the temporal component of this vector being proportional to $n_{\chi}-n_{\bar{\chi}}$. For the Majorana case only the axial vector $\bar{\chi} \gamma_{\mu} \gamma_{5} \chi$ remains and its temporal component becomes proportional to $n_{\chi_{l}}-n_{\chi_{r}}$, as in eq. (2).

The expression for the corresponding electron energy splitting is similar to that of eq. (3),

$$
\Delta E \sim d_{e}\left|\Delta n\left\langle\vec{\sigma}_{e} \cdot \vec{v}_{e}\right\rangle\right|
$$

where $\Delta n$ is the appropriate number density difference corresponding to the Dirac or the Majorana case. In principle these differences depend on the unknown nature of the dark matter and its evolution along the Universe history. In the following we will write these differences as $|\Delta n|=\alpha n$, where $n$ is the total WIMP number density. Clearly the parameter $\alpha$ is a measure of the degree of particle-antiparticle or left-right asymmetry present in the dark matter, respectively. For example, in the case of Dirac dark matter, $\alpha=1$ indicates that all WIMPs are particles with no antiparticles present and $\alpha=0$ means a complete particle-antiparticle symmetry. As in the neutrino case, interactions between molecular electrons with non-zero helicity and cold dark matter could lead to an energy difference between the two enantiomers of a chiral molecule whenever the parameter $\alpha$ is different from zero. To have an estimation of the energy difference, we consider the interaction between WIMPs and an electron of a chiral molecule. Let $\rho=n M_{\chi}$ be the energy density of those WIMPs, with $M_{\chi}$ being their mass and $n$ their number density. The density of WIMPs trapped in the gravitational potential wall of the galaxy is expected to be of the order of $\rho \sim 0.3 \mathrm{GeV} \mathrm{cm}^{-3}$. Then the energy splitting can be written as

$$
\Delta E \sim d_{e} \alpha \frac{\rho}{M_{\chi}}\left|\left\langle\vec{\sigma}_{e} \cdot \vec{v}_{e}\right\rangle\right|
$$

In order to see how important this splitting could be, we need to know which values of the coupling constant $d_{e}$ are acceptable. In principle there is no any available experimental information about $d_{e}$. However, one reasonable assumption that could be done is that all the $d_{i}$ couplings are at least of the same order of magnitude. In the absence of a theory of WIMPs this does not seem to be so bad an assumption since WIMPs does not interact strongly with matter. If this is the case, one can then use the present bounds on the elastic cross-section proton- $\chi$ to get some information about the size of the $d_{i}$ couplings. In order to compute this cross-section, one needs to relate the quark$\chi$ couplings with the proton- $\chi$ coupling. This can be done by using the effective Hamiltonian (see [34] and references therein)

$$
H=-a_{p} 2 \sqrt{2} \bar{\chi} \gamma_{\mu} \gamma_{5} \chi \bar{p} s^{\mu} p
$$

where $p$ is the proton spinor and $s^{\mu}$ is its spin vector (here we are considering the Majorana case but the Dirac 
case can be treated in a similar way). The coupling $a_{p}$ is defined as

$$
a_{p}=\frac{1}{\sqrt{2}} \sum_{i=u, d, s} d_{i} \Delta q_{i}^{(p)} .
$$

The constants $\Delta q_{i}^{(p)}$ (with $q_{1}=u, q_{2}=d$ and $q_{3}=s$ ) are introduced through the proton matrix element

$$
\left\langle p\left|\bar{\psi}_{i} \gamma^{\mu} \gamma_{5} \psi_{i}\right| p\right\rangle=2 s^{\mu} \Delta q^{(p)} .
$$

Experimentally we have $\Delta u^{(p)} \simeq 0.78, \quad \Delta d^{(p)} \simeq-0.5$ and $\Delta s^{(p)} \simeq-0.16$. Then, by using standard methods, it is straightforward to compute the elastic proton- $\chi$ cross-section, which is given in the proton rest frame by

$$
\frac{\mathrm{d} \sigma}{\mathrm{d} q^{2}}=\frac{\sigma_{n}}{4 v_{\chi} \mu^{2}}
$$

where $\vec{q}$ is the momentum transfer, $v_{\chi}$ is the $\chi$ velocity, $\mu$ is the proton- $\chi$ reduced mass and

$$
\sigma_{n}=\frac{12 a_{p}^{2} m_{p}^{2} M_{\chi}^{2}}{\pi\left(m_{p}+M_{\chi}\right)^{2}}
$$

( $m_{p}$ being the proton mass) is just the non-relativistic cross-section for vanishing momentum transfer. Nowadays there are many experiments around the world trying to detect WIMPs directly (visit the webpage in [35] for complete and upgraded report of their main results). Usually they set exclusion regions on the plane $\sigma_{n}-M_{\chi}$. From the recent XENON10 2007 [35] we learn, for example, that, for $M_{\chi} \simeq 100 \mathrm{GeV}, \sigma_{n}$ must be smaller than $10^{-43} \mathrm{~cm}^{2}$ and, for $M_{\chi} \simeq 1000 \mathrm{GeV}$, smaller than $10^{-42} \mathrm{~cm}^{2}$. Assuming for simplicity all the quark couplings to be the same, i.e. $d_{q} \simeq d_{u} \simeq d_{d} \simeq d_{s}$, we have $a_{p} \simeq 0.0072 d_{q}^{2}$. Then we get that for $M_{\chi} \simeq 100 \mathrm{GeV}, d_{q}^{2}<$ $10^{-14} \mathrm{GeV}^{-4}$ and for $M_{\chi} \simeq 1000 \mathrm{GeV}, d_{q}^{2}<10^{-13} \mathrm{GeV}^{-4}$. As discussed above we now assume $d_{e} \sim d_{q}$. Then it is possible to set a bound on the energy splitting which turns to be very tiny even in best case corresponding to $M_{\chi} \simeq 100 \mathrm{GeV}$. We obtain in this case, with an electron velocity about $10^{-2}, \Delta E \leqslant \alpha 10^{-44} \mathrm{eV}$.

Conclusion. - We have analyzed the effect of cold dark matter on the discrimination between the two enantiomers of a chiral molecule whose external electrons have opposite helicities in the respective opposite enantiomers. The estimated energy difference between the two enantiomers, due to WIMP-electron interaction, is found to be extremely small, several orders of magnitude lower than that induced by the electron-nuclei weak interaction. Hence, dark matter would be discarded as inductor of chiroselection between enantiomers and then of biological homochirality. By contrast, the enantioselective effect of the cosmological relic sea of neutrinos acquires relevance with the current assumptions about the number density of cosmological neutrinos. In this case we obtain an energy difference between $10^{-23}$ and $10^{-21} \mathrm{eV}$ for the two opposite enantiomers per molecular electron with non-zero helicity. The upper bound of the energy difference, although it could be excluded by reasons previously mentioned, reaches the same order of magnitude as the energy difference induced by the well-known electronnucleus electroweak interaction in some molecules.

$$
* * *
$$

This work is supported by the DGICYT (Spain) project BPA2005-02327, by the Universidad Complutense/CAM projects 910309 and CCG06-UCM/ESP-137, and by the MEC (Spain) projects CTQ2005-09185-C02-02 and FIS2004-03267. The work of PB was supported by the FPI grant BES-2006-11976 from the Spanish MEC. The authors would like to thank A. L. MARoto and R. PÉrez DE TUDELA for useful discussions.

\section{REFERENCES}

[1] Bonner W. A., Orig. Life Evol. Biosph., 21 (1991) 59.

[2] Jorissen A. and Cerf C., Orig. Life Evol. Biosph., 32 (2002) 129.

[3] Cline D. B. (Editor), The Physical Origin of Homochirality in Life, AIP Conf. Proc., 379 (1996).

[4] Hegstrom R. A., Rich A. and Van House J., Nature, 313 (1985) 391.

[5] Bailey J., Chrysostomou A. and Hough J. H. et al., Science, 281 (1998) 672.

[6] Bailey J., Orig. Life Evol. Biosph., 31 (2001) 167.

[7] Buschermhle M., Whittet D. C. B., Chrysostomou A. et al., Astrophys. J., 624 (2005) 821.

[8] Rikken G. L. J. A. and Raupach E., Nature, 405 (2000) 932.

[9] Ruchon T., Vallet M., Chauvat D. et al., J. Chem. Phys., 125 (2006) 084104.

[10] Engel M. H. and Macko S. A., Nature, 389 (1997) 265.

[11] Pizzarello S. and Cronin J. R., Geochim. Cosmochim. Acta, 64 (2000) 329.

[12] Bonner W. A., Orig. Life Evol. Biosph., 22 (1992) 407.

[13] Bouchiat A. M. and Bouchiat C. C., Rep. Prog. Phys., 60 (1997) 1351.

[14] Letokhov V. S., Phys. Lett. A, 53 (1975) 275.

[15] Rein D. W., Hegstrom R. A. and Sandars P. G. H., J. Chem. Phys., 73 (1980) 2329.

[16] Zanasi R., Lazzeretti A. and Soncini A., Phys. Rev. $E, 59$ (1999) 3382.

[17] Laerdahl J. K. and Schwerdtfeger P., Phys. Rev. Lett., 84 (2000) 3811.

[18] Soulard P. et al., Phys. Chem. Chem. Phys., 8 (2006) 79.

[19] Crassous J. et al., Org. Biomol. Chem., 3 (2005) 2218.

[20] Kondepudi D. K. and Nelson G. M., Nature, 314 (1985) 438.

[21] Plasson R., Kondepudi D. K., Bersini H., Commeyras A. and Asakura K., Chirality, 19 (2007) 589.

[22] Salam A., J. Mol. Evol., 33 (1991) 105.

[23] Avalos M., Babiano R., Cintas P., Jiménez J. L. and Palacios J. C., Tetrahedron Asymmetry, 11 (2000) 2845 . 
[24] Chandrasekhar S., Chirality, 20 (2008) 84.

[25] Bargueño P. and Gonzalo I., Orig. Life Evol. Biosph., 36 (2006) 171.

[26] Bargueño P. and PÉrez de Tudela R., Orig. Life Evol. Biosph., 37 (2007) 253.

[27] Muñoz C., Int. J. Mod. Phys. A, 19 (2004) 3093.

[28] Sadoulet B., Rev. Mod. Phys., 71 (1999) 197.

[29] Gelmini G. B., Phys. Scr., T121 (2005) 131.

[30] Stodolsky L., Phys. Rev. Lett., 34 (1975) 110.
[31] Pérez-Díaz J. L., PÉrez-García V. M. and Gonzalo I., Phys. Lett. A, 160 (1991) 453.

[32] Duda G., Gelmini G. and Nussinov S., Phys. Rev. D, 64 (2001) 122001.

[33] Dolgov A. D. et al., Nucl. Phys. B, 632 (2002) 363.

[34] Binetruy P., Supersymmetry: Theory Experiment and Cosmology (Oxford University Press) 2006.

[35] Dark Matter Tools Workgroup Home Page: http:// dmtools. berkeley.edu. 